\title{
Evaluation of Evidence on Radiographic Imaging and Clinical Examination Tests for Superior Labral Tear from Anterior to Posterior
}

\author{
Sydney M Baumgartner* \\ Department of Applied Medicine and Rehabilitation, LAT, ATC, USA
}

*Corresponding author: Sydney M Baumgartner, Department of Applied medicine and Rehabilitation, USA.

Received Date: September 14, 2019

Published Date: September 24, 2019

\section{Clinical Question}

Is radiographic imaging the best diagnostic technique available to identify a superior labral tear from anterior to posterior, when compared to clinical examination tests, in patients with concern for this shoulder pathology?

\section{Data Sources}

Google Scholar and PubMed databases were searched for SLAP tear, SLAP and meta-analysis in order to identify research between the ten-year span from 2007 to 2017.

\section{Study Selection}

Requirements for all articles included in this review, were that they needed to have record of sensitivity and specificity of a Superior Labral Tear from Anterior to Posterior in one or more of the following categories:

- Magnetic Resonance Imaging or Magnetic Resonance arthrography

- $\quad$ Active Compression/O'Brien's Test,

- Crank Test or Speed Test. Articles were excluded if the tests were not verified through functional anatomy or the patients were not being evaluated for a superior labral tear from anterior to posterior.

\section{Data Extraction}

The mean sensitivity and specificity for each test reviewed in the five different articles were organized by type of test preformed. All sensitivity and specificity scores were turned into decimals and rounded to the nearest hundredth and values were rounded up when warranted. The data included in this analysis is shown in (Table 1).
Table 1: Data Analyzed.

\begin{tabular}{|c|c|c|}
\hline Test, First Author (reference) year & Sensitivity & Specificity \\
\hline $\begin{array}{l}\text { Direct MR arthrography, Symanski, } \\
\qquad 2017\end{array}$ & 0.8 & 0.9 \\
\hline Direct MR arthrography, Connick, 2016 & $0.82-1.0$ & $0.71-0.98$ \\
\hline $\begin{array}{l}\text { Low-bias MR arthrography 3. Symanski, } \\
\qquad 2017\end{array}$ & 0.83 & 0.91 \\
\hline MR arthrography, Knesek, 2012 & 0.9 & 0.9 \\
\hline $\begin{array}{l}\text { SLAPII + direct MR arthrography } \\
\text { Symanski, } 2017\end{array}$ & 0.82 & 0.89 \\
\hline MR imaging, Symanski, 2017 & 0.63 & 0.87 \\
\hline 1.5-T MR imaging Symanski, 2017 & 0.81 & 0.67 \\
\hline 3.0-T MR imaging Symanski, 2017 & 0.78 & 0.99 \\
\hline Low-bias MR imaging Symanski, 2017 & 0.61 & 0.95 \\
\hline SLAPII + MR imaging Symanski, 2017 & 0.48 & 0.88 \\
\hline Active Compression Test, Knesek, 2012 & $0.47-1.00$ & 0.11-0.99 \\
\hline $\begin{array}{c}\text { Active Compression/ O’Brien's } \\
\text { Hegedus, } 2007\end{array}$ & 0.68 & 0.54 \\
\hline $\begin{array}{l}\text { Active Compression Test, Meserve, } \\
2007\end{array}$ & $0.47-0.78$ & $0.11-0.73$ \\
\hline Anterior Slide Test, Meserve, 2007 & $0.05-0.10$ & $0.82-0.93$ \\
\hline Crank Test, Meserve, 2007 & $0.13-0.58$ & $0.56-0.83$ \\
\hline Neer Test, Hegedus, 2007 & 0.12 & 0.96 \\
\hline Speed Test, Hegedus, 2007 & 0.32 & 0.61 \\
\hline Speed Test, Meserve, 2007 & $0.04-0.48$ & $0.67-0.99$ \\
\hline
\end{tabular}

\section{Main Results}

Of all the tests reviewed, the greatest specificity was found in the magnetic resonance arthrography with magnetic resonance imaging in a close second. Magnetic resonance imaging was recorded with the best sensitivity, followed by magnetic resonance 
arthrography, and Speeds Test in that order. A number of researchers indicated a moderate to high sensitivity and specificity in Active Compression/O'Brien's Test, Neer's and Speeds Test. These same researchers often cautioned against the use of these tests.

\section{Summary}

Superior labral tears from anterior to posterior (SLAP), is a tear in from ten- to two o'clock in the labrum of the glenohumeral joint. A prevalence of six- to twenty-six percent of people who have undergone arthroscopic repair on their shoulder have a SLAP tear [1]. Incidence of this pathology is growing as repetitive training in overhead activities is becoming increasingly popular and intense among today's youth [1]. SLAP tears are broken into four different categories based on their severity and further diagnosed as one of ten types [1]. Even with the prevailing research that has been conducted on SLAP tears, there is still question on a decisive way to correctly diagnose them [2]. Identification for these lesions in the clinic is difficult due to the lack of a pattern in symptoms and clinical examination test scores [3]. Evidence has shown that imaging is the greatest way to identify a SLAP tear.

Symanski Js, et al. [3] reviewed research on magnetic resonance imaging (MRI) and magnetic resonance arthrography (MRA) and found that MRA had the greatest sensitivity of all tests evaluated, with high reliance in ruling out a Type II SLAP Lesion. Another study also reported the high to perfect sensitivity of direct MRA [4]. The greatest specificity was found in the 3.0-T MRI with low-bias MRI not far behind [3]. Another study identified the specificity of direct MRA to span from moderate to high [2].

MRA is able to rule out a SLAP lesion due to the enhanced clarity provided by the dye injected into the joint prior to the procedure. This define the location of where an anatomically correct labrum would be attached and its absence in the case of a SLAP tear [3]. In the general population, contrast is also needed in chronic cases of shoulder pain, as it is better able to identify SLAP tears that did not produce symptoms in the clinical evaluation. Acute injury evaluations benefit from the use of MRA as this technique allows the clinician to identify malformation in the soft tissues and effusion within the joint [4]. MRA is also effective in identifying micro-tears in the labrum, that may lead to SLAP lesions, in first-class athletes who are experiencing symptoms of overuse [3].

Even though these tests are effective at identifying a SLAP Tear, they do hazard the patient with radiation [3]. Therefore, clinical special tests have been assessed for their ability to diagnose the condition [4]. Imaging is not always correct, and a high prevalence of false positives have been reported [1]. It is also common for a false positive to occur, as normal variances are a part of the anatomy of the glenohumeral joint, especially associated with the labrum, and are often labeled as a SLAP lesion.

Clinical special tests are convenient for medical professionals to perform in virtually any environment and omit the need for the large expensive equipment needed when utilizing medical imaging. The Active Compression/O'Brien's Test was recorded with the highest sensitivity and specificity in two separate articles [1,5]. Hegedus EJ, et al. [2] deemed Neer and Speed Tests unable to identify a SLAP lesion. However, a group of researchers reported the Speeds Test to have a specificity that ranges from moderate to high, making it comparable to the sensitivity of imaging techniques [5].

This variability in data reveals the difficulty in the ability to develop a clinical evaluation pattern effective for identifying a SLAP lesion. In order to try and investigate this issue further, clinical examination tests have been paired during evaluations and the same inconsistent results as before surface [1]. The patient's history may also be a vital part in the diagnosis of a SLAP tear, although not every case includes the same symptoms. Kinematics can also be evaluated during movement of the shoulder girdle, in order to identify any abnormalities, such as scapular dyskinesia, that may be a predisposing factor for a labral tear.1 Evidence is lacking in the ability to effectively evaluate the reliability of these clinical examination tests.

The conclusion drawn from evidence evaluated by the author of this review promotes the use of MRA as the final evaluative test to diagnose a SLAP lesion due to its high diagnostic accuracy, prevalent among literature. Clinical examination tests such as Active Compression/O'Brien's, Speeds and Neer's test have been reported as reliable in a variety of literature but is also cautioned as unreliable in a high number of articles. Imaging is consistently reported as an effective way to diagnose a SLAP lesion in all of the research that has been conducted on this technique. Therefore, clinical examination tests are an acceptable way to create a differential diagnosis of a SLAP lesion, but MRA is ultimately the nonpareil way to detect a SLAP tear.

\section{Acknowledgment}

None.

\section{Conflicts of Interest}

No conflict of interest

\section{References}

1. Knesek M, Skendzel JG, Dines JS, Altchek DW, Allen AA, et al. (2012) Diagnosis and Management of Superior Labral Anterior Posterior Tears in Throwing Athletes. Am J Sports Med 41(2): 444-460.

2. Hegedus EJ, Goode A, Morin A, Tamaddon M, Moorman CT, et al. (2008) Physical examination tests of the shoulder: a systematic review with meta-analysis of individual tests. Br J Sports Med 42(2): 80-92.

3. Symanski JS, Subhas N, Babb J, Nicholson J, Gyftopoulos S (2017) Diagnosis of Superior Labrum Anterior-to-Posterior Tears by Using MR Imaging and MR Arthrography: A Systematic Review and Meta-Analysis. Radiology 285(1): 101-113.

4. Coninck TD, Ngai SS, Tafur M, Chung CB (2016) Imaging the Glenoid Labrum and Labral Tears. Radiographics 36(6):1628-1647.

5. Meserve BB, Cleland JA, Boucher TR (2009) A meta-analysis examining clinical test utility for assessing superior labral anterior posterior lesions. Am J Sports Med 37(11): 2252-2258. 